\title{
Structural Design of a CNC Router Based on Optimization Technique
}

\author{
Nguyen Huu Loc ${ }^{1,2, ~ *, ~ T r a n ~ V a n ~ T h u y ~}{ }^{1,2}$ \\ ${ }^{1}$ Faculty of Mechanical Engineering, Ho Chi Minh City University of Technology (HCMUT), Ho Chi Minh City, Vietnam \\ ${ }^{2}$ VietNam National University of Ho Chi Minh City (VNUHCM), Ho Chi Minh City, Vietnam
}

Email address:

nhloc@hcmut.edu.vn (N. H. Loc), tvthuy@pvd.edu.vn (T. V. Thuy)

${ }^{*}$ Corresponding author

\section{To cite this article:}

Nguyen Huu Loc, Tran Van Thuy. Structural Design of a CNC Router Based on Optimization Technique. International Journal of Mechanical Engineering and Applications. Vol. 8, No. 3, 2020, pp. 81-87. doi: 10.11648/j.jimea.20200803.11

Received: May 17, 2020; Accepted: May 29, 2020; Published: June 17, 2020

\begin{abstract}
Optimizations are a powerful tool for engineers as well as product designers. Using optimization helps us to find out new concept designs that satisfy the requirements of functions, aestheticism, material, cost, and time. This paper gives an overview of structural optimization and its effects on the design process, provides the process and application of the topology optimization as well as its ability and importance in design to improve product and product design processes in general and $\mathrm{CNC}$ router in particular. Application of optimization methods includes topology, shape, and size optimization to design the structures of the $\mathrm{CNC}$ router. The results have built the optimization design process of CNC router structure, secured material reduction, as well as improved shape and size of $\mathrm{CNC}$ router structure. In the framework of this paper, the high applicability of structural optimization in the design of the CNC router structure will be properly introduced. Simulation for structural design and analysis has developed according to the strong and widespread application of computers and flexible application of $\mathrm{CAD} / \mathrm{CAID} / \mathrm{CAE}$ software in design has proven superior advantages compared to previous methods. The obtained results not only ensure the technical and aesthetic requirements of the design but also reduce the material.
\end{abstract}

Keywords: Optimization, Structural Design, CNC Router, CAD/CAE Systems

\section{Introduction}

In order to survive in the market economy, products must meet the three decisive factors: the highest quality, the lowest cost, and the shortest time to be launched into the market. According to this demand, more and more design support software (CAD) and structural analyses (CAE) with optimal functions have been integrated into the design process. Galileo was the first person to study the optimization problem in mechanics. After that, optimization algorithms were continuously developed based on standard functions such as Bernoulli, Euler, Lagrange. Especially, Cauchy was the one who made significant contributions when making algorithms for gradient method in the year $1847[1,2]$. Numerical methods combined with mathematical programming methods to solve the optimal algorithms were studied in 1960. Since then, the development of structural optimization has been associated with the evolution of electronic computers. The extension of the concept of optimal shape design for mechanical structures was implemented in the 1970s. Haftka and Grandhi introduced shape optimization with design variables as node coordinates of the structural frame or node coordinates of spline control points surrounding the structure [1].

In the late $80 \mathrm{~s}$, topology optimization was studied. Since then, structural optimization has developed strongly with many researchers in this field, and many pieces of CAD and CAE software have appeared. Until the 1990s, the combination of $\mathrm{CAD}$ and CAE became a major factor in reducing time and eliminating several stages of the design cycle and optimization of applications in production. The automotive and aviation industry invests primarily in optimal research and technical applications. From 2000 to present, CAE completely replaced physical testing. Because of its novelty, despite being accepted as a useful design tool, optimization of the structure has not been commonly used in industry [3].

Thus, simulation for structural design and analysis has 
developed according to the strong and widespread application of computers. The simulation of dynamic and complex structural dynamics analysis will increase in the future [4-6]. With system modeling and simulation, we can observe its movement and the complex interactions between the elements in the system. However, this model is based on data and results of calculations, observations, measurements rather than optimal results. By incorporating optimization into modeling, the designer not only eliminates many of its limitations but also extends to more areas of application and research.

Today, many pieces of CAD / CAE software have developed topological design optimization systems, such as ANSYS, Hyperworks, Autodesk Inventor, Solidworks, and Fusion 360.

\section{Theoretical Background}

\subsection{Topology Optimization}

\subsubsection{Topology Optimization Method}

The basis of the topology optimization process is to find the distribution of materials in a given area called the design space, so that the structure can fullfil the requirements of load, vibration frequency, and satisfy the constraints of mass, volume, and fabrication capacity. Many methods of finding the optimal structure have been developed, such as the homogeneous method, density method, and evolution method.

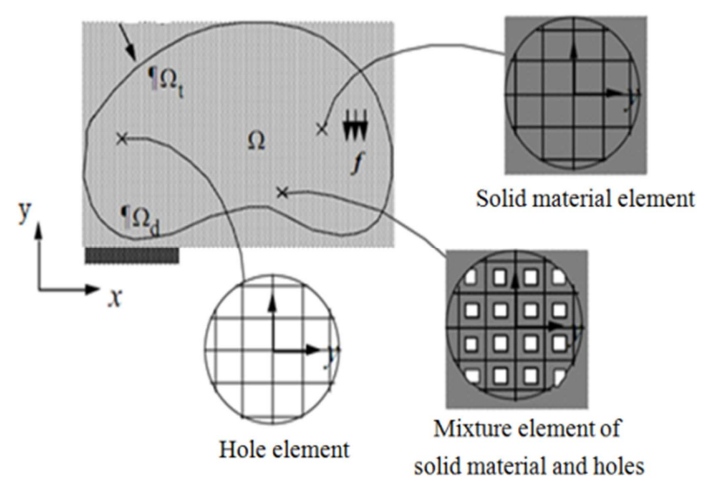

Figure 1. The material model according to the homogeneous method [7].

1. The homogeneous method uses a composite material model, as shown in Figure 1, in which the material at an element is considered to be a mixture of solid material (density equal to 1) and holes (density equal to 0 ). Each element is a square with a side length of 1 unit with an inner rectangular hole. The design variable is the size of a rectangular hole $\alpha$ and $\beta$ (varies from 0 to 1) [7].

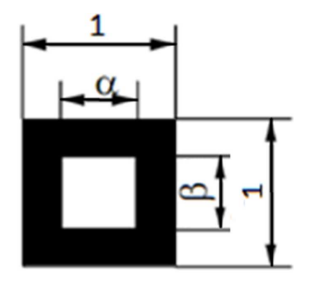

Figure 2. The size of holes $\alpha$ and $\beta$ in the element.
With this material model, the problem of finding material distribution is transformed into the problem of optimizing the hole with size $\alpha \times \beta$ in each element in Figure 2.

2. Density method: Given by R. J. Yang and B. P. Wang since 1994, this method uses an isotropic, artificial material model with different density between elements. The advantage of this method includes simplicity, fast convergence speed, and small calculated volume [8],

3. Evolution method: This method does not use design variables as above. The design space will be separated into each element. The evolutionary algorithm will take into account the stress level in each element and remove the elements that do not meet the required stress level. After each time the elements are removed, the stress in the structure will change, and the elements with small stresses will continue to be eliminated. If this method converges, it will create a structure in which the stresses in the elements are approximately equal and close to the maximum stress. However, some studies have shown that this method can create non-optimal structures, especially if the standard of hardness is taken into consideration [7].

Currently, commercial CAE software such as MSC Patran-Nastran and ANSYS / Shape Optimization makes calculation based on density method [9]. The software employs an isotropic material model and uses only one design variable, which is the density of the material. The structure is separated into elements by the finite element method. Because the density of each element varies, the Young elastic modulus of each element is also different. Characteristic of the density of each element is the density coefficient $x$.

$$
x_{e}=\frac{\rho_{e}}{\rho_{o}}
$$

If the material is consistent across the whole element, the elastic module can be calculated using the exponential rule, as shown in Figure 3:

$$
E_{e}=x_{e}^{p} E_{o}
$$

The compensation value $\mathrm{p}$ is given to make the design variable between 0 and 1 when $p \geq 1$, usually $p$ has a value between 2 and 4 [8].

The method of density optimization is based on structural hardness standards. With the structural hardness found, it will reach the maximum value corresponding to the amount of material used. Where $c(x)$ is the deformation energy of each element with a density coefficient, and can be stated as follows:

$$
\begin{aligned}
& c(x)=f^{T} u \\
& f=K(x) u
\end{aligned}
$$

where $K(x)$ is the element stiffness matrix with material density $\mathrm{x}$. 


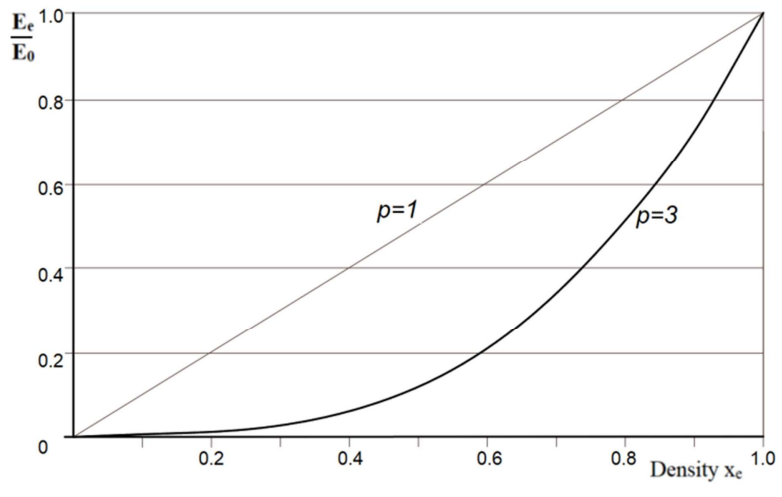

Figure 3. The modulus of elasticity of the element has density $x_{e}[8]$.

Since the deformation energy is inversely proportional to the element hardness, the hardness is most significant when the deformation energy reaches the minimum value:

$$
\min : c(x)=\sum_{e=1}^{N} u_{e}^{T} K_{e}\left(x_{e}\right) u_{e}
$$

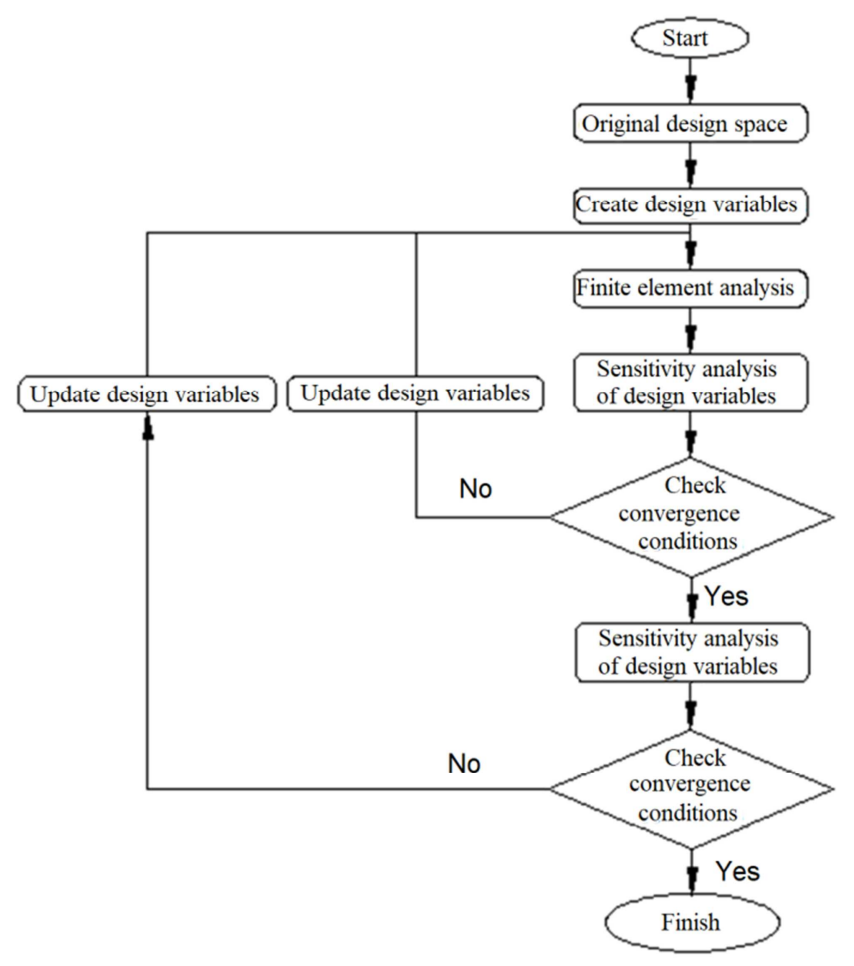

Figure 4. Topology optimization calculation process.

The optimal problem must also satisfy the constraints, i.e. during the calculation process, the mass of the structure is constant:

$$
h(x)=\sum_{e=1}^{N} V_{e} \rho_{e}-M_{0}=0
$$

and $0 \leq \rho_{\min } \leq \rho_{e} \leq 1$

\subsubsection{Topology Optimization Calculation Process}

Topology optimization software works with an input file containing commands to be executed to solve the problem (SOL-command). The input file also includes decks containing parameters of the geometric model of structure, mesh, support, impact force, etc... and cards containing information for each parameter such as force set point, the magnitude of force, etc... When the software starts running, the SOL commands will be called; the SOL command sets a suitable sequence for the executable commands $[8,10,11]$.

After each calculation, the optimal algorithm will create a new structure which will be tested by the conditions of convergence. If the result is satisfied, the calculation will stop and an optimal structure will be obtained.

\subsection{Topology and Size Optimization}

Size optimization is the simplest solution because it only modifies the size characteristics of the design. Size optimization began to be studied by Schmit since 1960 and has been in use since the 1970s in SAMCEF and ELFIN software. This method is often utilized to optimize the frame structure, in which the size change usually invloves the length and section of the bars, the arrangement of the structure remains unchanged. Topology and size optimization process shown in Figure 5.
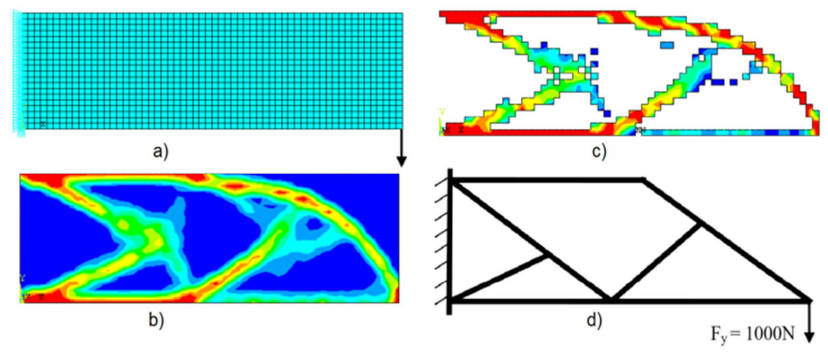

Figure 5. The topology and size optimization process

Shaping optimization methods were developed by Zolesio (1981) and Haug (1986) at the University of Iowa. In such methods, design variables define detailed shape such as radius of curvature, the position of nodes in a finite element model, or position of control points of Bezier curves, BSpline, as shown in Figure 6. In the process of size optimization, the finite element model remains unchanged. Size optimization is just a separate case of shape optimization.

In CAE software, the shape and size optimization process is carried out by the Design of Experiment method (DOE). Accordingly, the program will take samples of parameter values alternately, then calculate, compare the obtained results and choose the optimal value for the parameters.

Regarding the optimization problem of shape and size, there are usually these following components:

Design variable (parameters which affect the shape and size of details)

Constraints (requirements which must meet the details such as maximum stress, stiffness, safety factor, specific vibration frequency, etc.)

Optimal objective (usually the minimum of mass - the part that consumes the least material). 


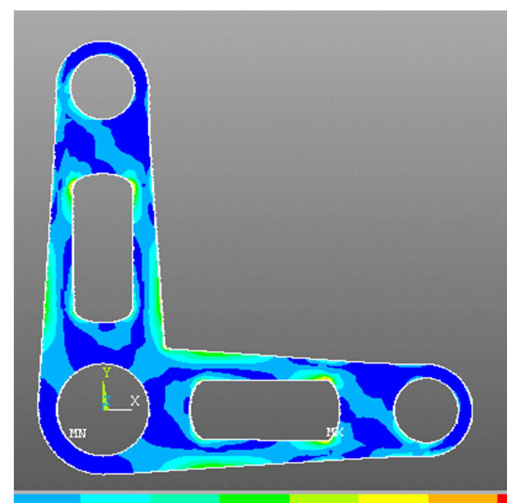

a) Before

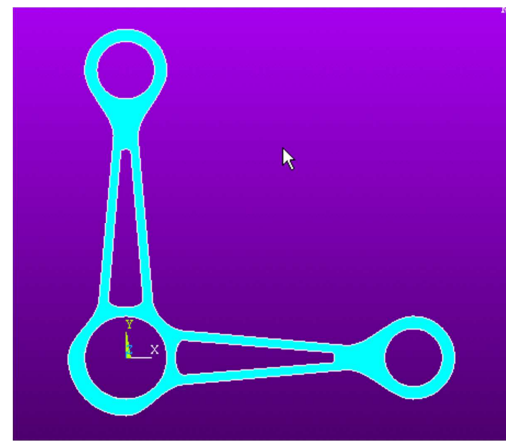

b) After

Figure 6. The shape and size optimization method.

The general optimization problem can be stated as follows $[14,15]$ :

$$
\text { Find } X=\left\{\begin{array}{c}
d_{1} \\
d_{2} \\
\vdots \\
d_{n}
\end{array}\right\} \text { to minimize } \mathrm{f}(\mathrm{X})
$$

Subject to:

$$
\begin{array}{r}
h_{j}(X) \leq 0 ; j=1,2, \ldots, n_{j} \\
l_{k}(X)=0 ; k=1,2, \ldots, n_{k} \\
d_{i l} \leq d_{i} \leq d_{i u} ; i=1,2, \ldots, n
\end{array}
$$

where $\mathrm{d}_{\mathrm{i}}$ - design variable; $\mathrm{X}$ - vectors of design variables; $\mathrm{f}$ objective function; $h_{j}$ - inequality function; $l_{k}$ - equality function; $\mathrm{n}$ - design variable number; $\mathrm{n}_{\mathrm{j}}$ - number of inequality functions; $\mathrm{n}_{\mathrm{k}}$ - number of equation functions.

\section{Numerical Result}

In Figure 7, the researcher constructs a hypothesis that the body is composed of four elements linked together, such as the body head (1), the body beam (2), the body column (3), and the body base (4). In order to symplify the calculations and ensure no loss of generality, it is realized that the body head and the body column are a cantilever beam corresponding to the length $\mathrm{L}_{1}, \mathrm{~L}_{3}$, and the body beam and the body base are beams corresponding to the length $\mathrm{L}_{2}$ and $\mathrm{L}_{4}$. Thus, the optimal problem for $\mathrm{CNC}$ milling machine body is equivalent to that of 4 elements (1), (2), (3), and (4).

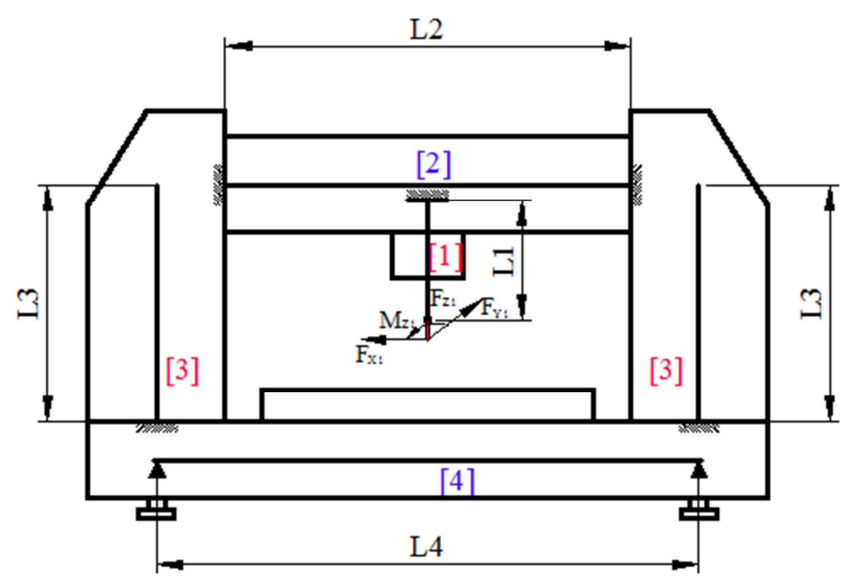

Figure 7. Wood machining CNC machine model.

\subsection{Shape Optimization}

Separate the model (Figure 7) into the elements (1), (2), and (3), and convert the model into a simple form, and set the force conditions is shown in Figure 8.
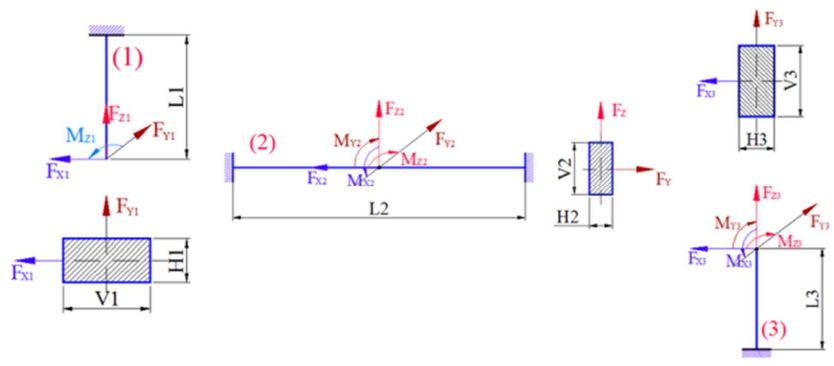

Figure 8. Force components act on machine elements.

After the preliminary design, the modeling of elements (1), (2), (3) is performed into the simulation environment, and optimization is made using CAE software. Optimal results are shown in Figures 9, 10, and 11.
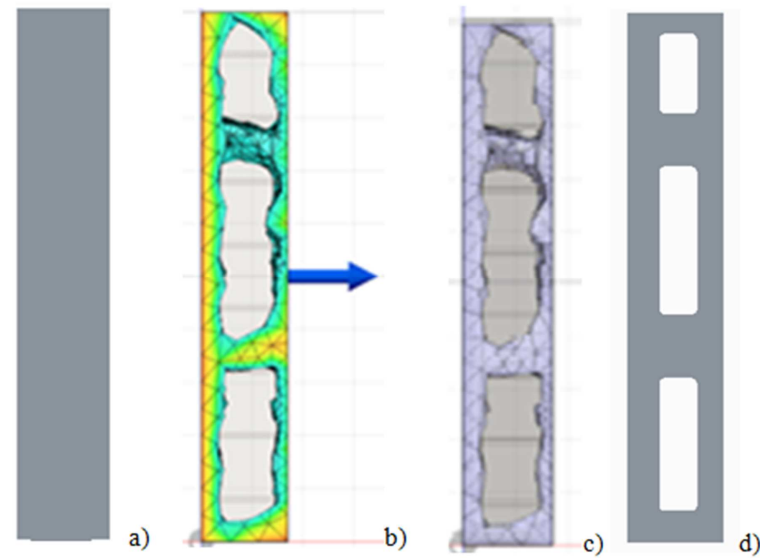

Figure 9. a) Initial model; b) Stress analysis; c) Topology optimization; d) Redesign. 
a)

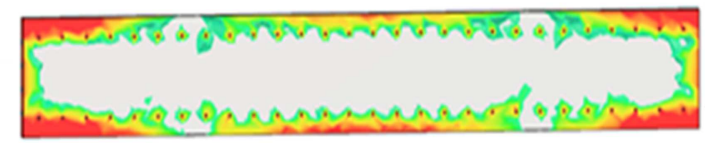

b)

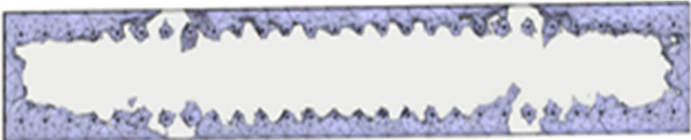

c)

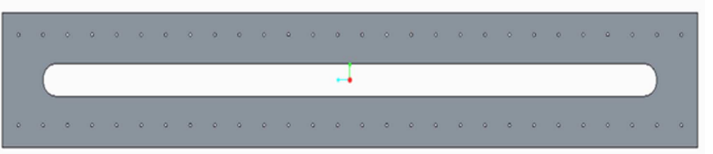

Figure 10. a) Stress analysis; b) Topology optimization; c) Redesign.

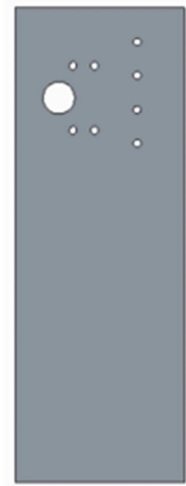

a)

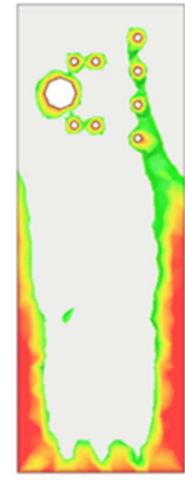

b)

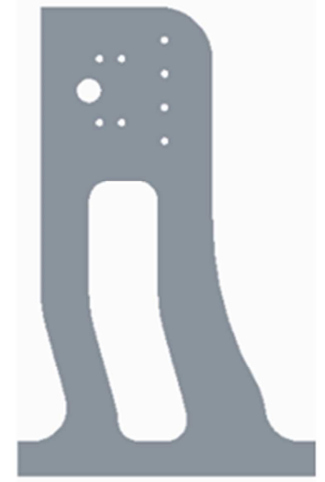

c)
Figure 11. a) Preliminary design of column structure; b) Stress analysis; c) Redesign.

\subsection{Size Optimization}

Parameters and optimization objectives: In this section, the gantry structure of the $\mathrm{CNC}$ machine, as shown in Figure 12 , is optimized by using the multi-objective genetic algorithm (MOGA) method to improve many goals of the machine structure. Besides, a sensitivity analysis is conducted to determine the influence of the initial parameters on the output parameters. After determining the most useful parameters, MOGA is implemented utilizing the Exploration Design toolbox of ANSYS so as to achieve the most optimal solutions.

The lower and upper limit dimension values (minimum and maximum) for each parameter variable are determined and shown in Table 1.

Table 1. Lower and upper limits size of the parameters.

\begin{tabular}{lllll}
\hline No. & $\begin{array}{l}\text { Dimension } \\
\text { Parameter }\end{array}$ & $\begin{array}{l}\text { Base } \\
\text { Dimension }\end{array}$ & $\begin{array}{l}\text { Lower } \\
\text { bound }\end{array}$ & $\begin{array}{l}\text { Upper } \\
\text { bound }\end{array}$ \\
\hline 1 & V1 & 140 & 126 & 154 \\
2 & V2 & 139.5 & 125.53 & 153.42 \\
3 & V3 & 50 & 45 & 55 \\
4 & V4 & 210 & 189 & 231 \\
5 & H1 & 270 & 243 & 297 \\
6 & W1 & 30 & 27 & 33 \\
7 & W2 & 20 & 18 & 22 \\
8 & A & 145 & 130.5 & 159.5 \\
\hline
\end{tabular}
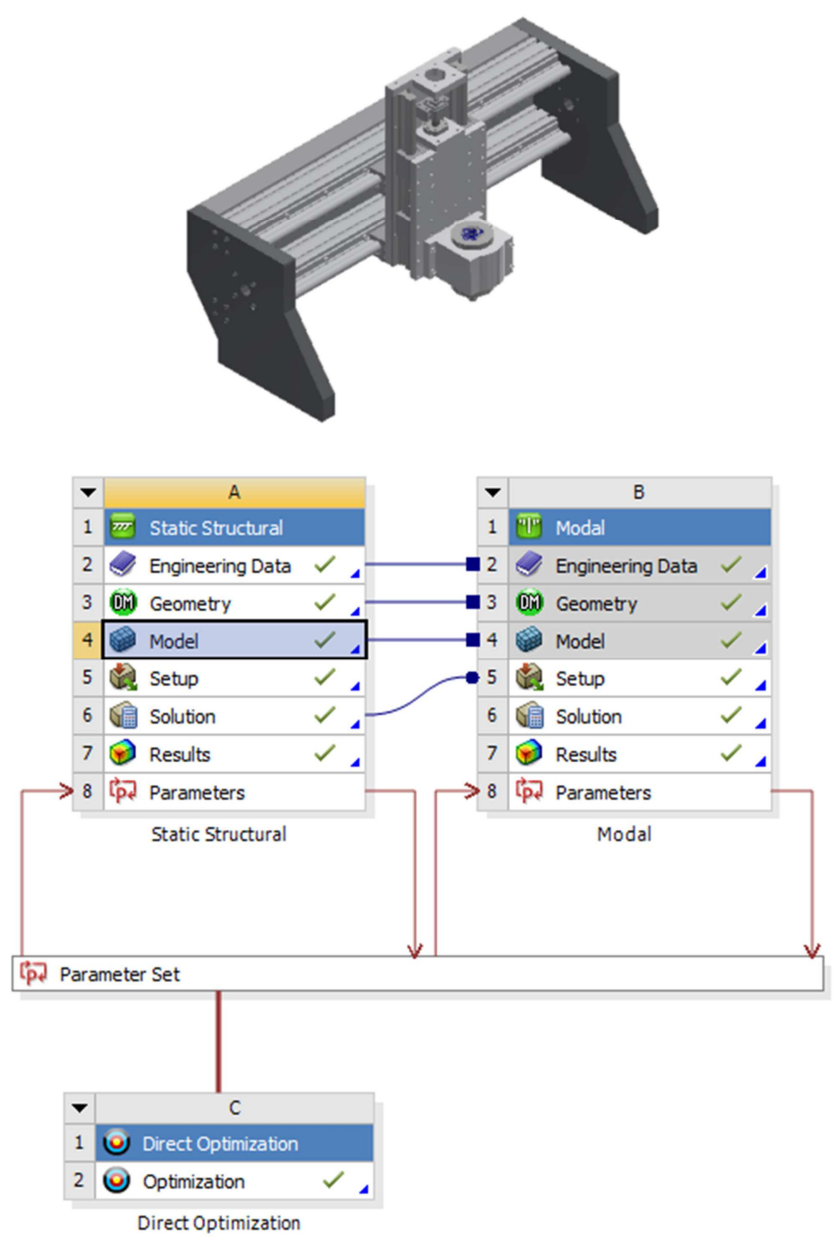

Figure 12. Gantry structure of $C N C$ machine and optimal diagram in ANSYS.

The input parameters for the optimization process are the basic dimensions of the gantry structure shown in Figure 13.
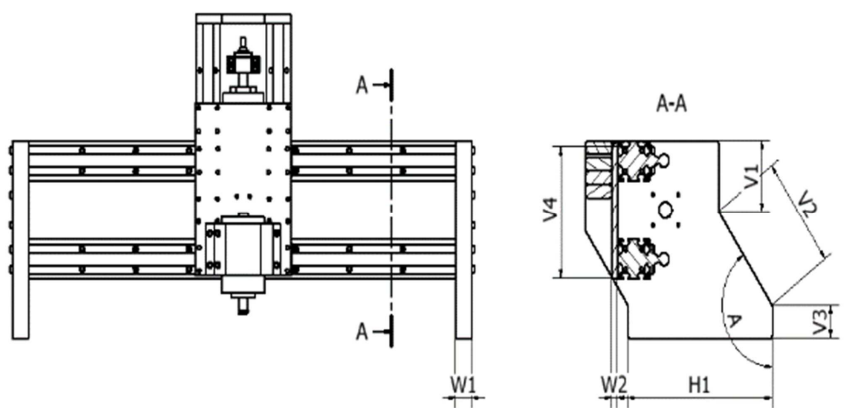

Figure 13. The basic dimensions of gantry structure.

The five optimization objectives include the first three natural frequencies of gantry structure related to $\mathrm{CNC}$ machine dynamic characteristics, displacement of the spindle head, and the weight of the gantry structure. The gantry volume and displacement of the spindle must be minimized while the natural frequency of the machine must be maximized.

\subsection{Optimized Dimension Results}

After optimization, the results are illustrated in Table 2. 
Table 2. Optimal results.

\begin{tabular}{lllll}
\hline No. & Input parameter & Symbol & Before Optimiza- tion & After Optimiza-tion \\
\hline 1 & & $\mathrm{~V} 1$ & 140 & 129.28 \\
2 & & $\mathrm{~V} 2$ & 140 & 133.84 \\
3 & & $\mathrm{~V} 3$ & 50 & 46.151 \\
4 & $\mathrm{~V} 4$ & 210 & 199.79 \\
5 & The dimension of gantry structure & $\mathrm{H} 1$ & 270 & 261.17 \\
6 & $\mathrm{~W} 1$ & 30 & 29.915 \\
7 & $\mathrm{~W} 2$ & 20 & 21.419 \\
8 & & $\mathrm{~A}$ & & 151.04 \\
\hline & & & 269.68 \\
\hline & & $\mathrm{f} 1$ & 250.64 & 374.55 \\
\hline 1 & & $\mathrm{f} 2$ & 339.58 & 447.7 \\
2 & Optimal Parameter & $\mathrm{f} 3$ & 405.02 & $7.044 \times 10^{-5}$ \\
3 & Natural frequency mode 1 & $\mathrm{u}$ & $8.215 \times 10^{-5} 140.7$ & 136.58 \\
4 & Natural frequency mode 2 & $\mathrm{m}$ & & \\
5 & Spindle displacement, $\mathrm{mm}$ & & & \\
\hline
\end{tabular}

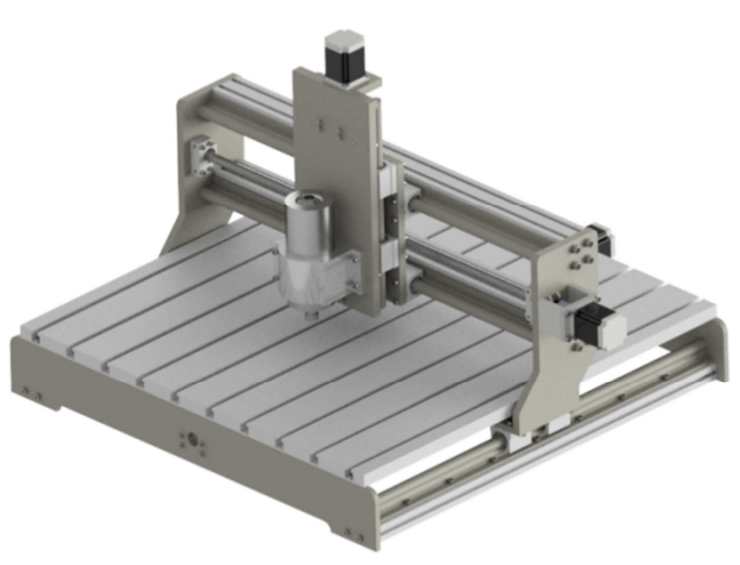

Figure 14. Complete modeling of machines before optimization.

Following the process of optimizing the details of the machine, a complete machine will be built before and after optimization as shown in Figures 14, 15.

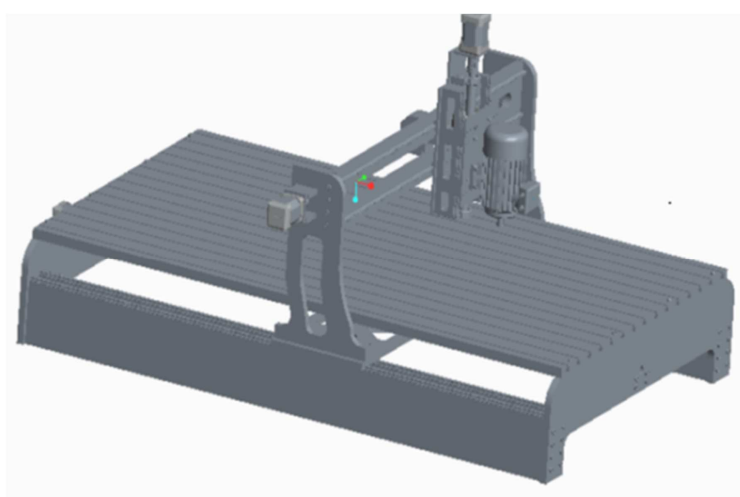

Figure 15. Complete modeling of machines with optimized elements.

\section{Conclusions}

Currently, the life cycle of a product is very short, so a good product must not only meet the needs of users but also have high aesthetics, diverse and abundant models, and the capacity to quickly change so as to satisfy market demands. Flexible application of CAD/CAID/CAE software in design has proven superior advantages compared to previous methods.

This paper presents the process of design optimization in a wood CNC machine structure. It also looks at the ideas of topology and size optimization, as well as its ability and importance in design to improve the shape and size of products and complete the process of product design, create product models to meet the increasing demands of customers. Topology optimization on $\mathrm{CAD} / \mathrm{CAE}$ systems helps us reduce materials, obtain the product shape of machine structures and machine parts in a short time, while our predecessors had to spend several decades on utilization, exploration, and improvement.

The achieved results confirm the tremendous potential of optimization in product design, material savings, and can be applied to many different machine structures and mechanical systems.

Structural optimization has beneficial applications in the design process. The ability of structural optimization can go even further in the future as more and more people participate in research and use design support software.

\section{References}

[1] S. Seireg, Optimizing the shape of mechanical elements and structures vol. 105: CRC Press, 1997.

[2] Y. Wang, H. Xu, and D. Pasini, "Multiscale isogeometric topology optimization for lattice materials," Computer Methods in Applied Mechanics and Engineering, vol. 316, pp. 568-585, 2017.

[3] K. Saitou, K. Izui, S. Nishiwaki, and P. Papalambros, "A survey of structural optimization in mechanical product development," Journal of computing and information science in engineering, vol. 5, pp. 214-226, 2005.

[4] S. Ananiev, "On equivalence between optimality criteria and projected gradient methods with application to topology optimization problem," Multibody System Dynamics, vol. 13, pp. 25-38, 2005.

[5] E. Tekin and I. Sabuncuoglu, "Simulation optimization: A comprehensive review on theory and applications," IIE transactions, vol. 36, pp. 1067-1081, 2004. 
[6] U. Schramm and M. Arold, "Optimization technology in design: trends, direction, and gaps," in Modeling and Simulation for Military Applications, 2006, p. 62280I.

[7] M. P. Bendsøe and N. Kikuchi, "Generating optimal topologies in structural design using a homogenization method," Computer methods in applied mechanics and engineering, vol. 71, pp. 197-224, 1988.

[8] M. P. Bendsøe, Topology optimization: Springer, 2009.

[9] R. Yang and A. Chahande, "Automotive applications of topology optimization," Structural optimization, vol. 9, pp. 245-249, 1995.

[10] D. Cholaseuk, "A stress-based material distribution method for optimum shape design of mechanical parts," Science \& Technology Asia, pp. 17-21, 2006.

[11] J. Wu, N. Aage, R. Westermann, and O. Sigmund, "Infill optimization for additive manufacturing - approaching bonelike porous structures," IEEE transactions on visualization and computer graphics, vol. 24, pp. 1127-1140, 2017.

[12] G. Allaire, F. De Gournay, F. Jouve, and A.-M. Toader, "Structural optimization using topological and shape sensitivity via a level set method," Control and cybernetics, vol. 34, p. 59, 2005.

[13] J. Wu, C. C. Wang, X. Zhang, and R. Westermann, "Selfsupporting rhombic infill structures for additive manufacturing," Computer-Aided Design, vol. 80, pp. 32-42, 2016.

[14] Nguyen Huu Loc, N. Y. Nguyen, Structural optimization design. National University publisher HCMC: Viet Nam, 2018.

[15] T. Wu, K. Liu, and A. Tovar, "Multiphase topology optimization of lattice injection molds," Computers \& Structures, vol. 192, pp. 71-82, 2017. 